\title{
Improving the Compatibility of Biodegradable Poly (Lactic Acid) Toughening with Thermoplastic Polyurethane (TPU) and Compatibilized Meltblown Nonwoven
}

\author{
Md Obaidur Rahman* ${ }^{*}$, Feichao Zhu, Bin Yu \\ College of Textile Science and Engineering, Zhejiang Provincial Key Laboratory of Industrial Textile Materials and Manufacturing \\ Technology, Zhejiang Sci-Tech University, Hangzhou, China \\ Email: ^bdobaidurrahman@gmail.com
}

How to cite this paper: Rahman, M.O., Zhu, F.C. and Yu, B. (2022) Improving the Compatibility of Biodegradable Poly (Lactic Acid) Toughening with Thermoplastic Polyurethane (TPU) and Compatibilized Meltblown Nonwoven. Open Journal of Composite Materials, 12, 1-15.

https://doi.org/10.4236/ojcm.2022.121001

Received: September 12, 2021

Accepted: December 13, 2021

Published: December 16, 2021

Copyright $\odot 2022$ by author(s) and Scientific Research Publishing Inc. This work is licensed under the Creative Commons Attribution International License (CC BY 4.0).

http://creativecommons.org/licenses/by/4.0/

(c) (i) Open Access

\begin{abstract}
Poly (Lactic Acid) (PLA) is a biodegradable polymer which originates from natural resources such as corn and starch, offering excellent strength, biodegradability, nevertheless its inherent brittleness and low impact resistance properties have limited its application. On the other hand, Thermoplastic Polyurethane (TPU) has high toughness, durability and flexibility, which is one of the most potential alternatives for enhancing the flexibility and mechanical strength of Poly (Lactic Acid) (PLA) by blending it with a compatibilizer. With the aim to improve the mechanical and thermal properties of Poly (Lactic Acid) (PLA) meltblown nonwovens, The Thermoplastic Polyurethane (TPU) was melt blended with Poly (Lactic Acid) (PLA) at the different corresponding proportions for toughening the Poly (Lactic Acid) and the corresponding PLA/TPU MBs (meltblown nonwovens) were also manufactured. Joncryl ADR 4400 is mixed into the PLA matrix during processing. It was found that Joncryl had a much higher chain extension that substantially increased the molecular weight of the PLA matrix. SEM study revealed that Joncryl ADR 4400 is a good compatibilizer. Moreover, in this study, the crystallization, thermal and rheological behaviors of the corresponding PLA and TPU blends were also investigated. PLA/TPU MBs were also characterized by morphology and mechanical properties. The rheological property of the PLA/TPU meltblown nonwoven revealed that the viscosity is increasing as the amount of TPU is increasing in the blend, PLA/TPU meltblown nonwovens exhibited excellent mechanical properties; they are soft, elastic, and have certain tensile strength. New materials have potential applications in the medical and agricultural fields. Joncryl ADR 4400 compatibilized blends showed higher strength than simple PLA/TPU blends at the same PLA/TPU ratio.
\end{abstract}




\section{Keywords}

Poly (Lactic Acid), Thermoplastic Polyurethane, Meltblown, Toughening, Nonwoven

\section{Introduction}

Day by day, the demand for bio-based and biodegradable polymers products is increasing in all productions areas for reducing the high amount of carbon emission, ensuring environmental sustainability and also for waste management. During the whole period of the COVID-19 pandemic, it has been seen that the use of PP and other petroleum-based materials masks has been increased at an alarming rate which is not fully environmentally friendly and also does not maintain the mask recycling system in many countries. In order to stop spreading the COVID-19 virus and to protect people from all over the world used masks for life safety.

In this paper, PLA toughened super meltblown fabric has been introduced which has high mechanical and antibacterial properties. Meltblown nonwoven is an industrialized simple, unique and one-step process whereas polymer resins are heated in an extruder and then extruded through an orifice die tip, while the stream is drawn by high-velocity hot air onto a collector screen to form a finely fibrous and self-bonding web for producing superfine diameter fibers ( $1-5 \mathrm{um}$ ); in the form of a micrometer or nanoscale [1] [2] [3]. In recent years, for producing the nonwoven fabrics using melt blown technology, there has been considerable interest grown up in the melt blowing process from both commercial and scientific viewpoints [4] and the Polyethylene (PE), Polypropylene (PP), Poly (Butylene Terephthalate) (PBT) [5], Thermoplastic Polyurethane (TPU) [6], Polyamides (e.g., nylon), Polystyrene (PS), poly (methyl methacrylate), and Poly (Lactic Acid) (PLA) have drawn considerable interest in this sector for manufacturing biodegradable nonwoven products.

With the improvement of research in biomedical applications such as tissue engineering, scaffolds and fracture fixation devices even PLA can replace stainless steel and metallic medical devices [7] [8] [9]. PLA MBs nonwoven is a good candidate for replacing non-biodegradable and petroleum-based polymer, and it also shows potential finance in the near future [10]. To improve the inherent drawbacks of PLA such as low thermal stability, crystallization speed, and ductility, compatibilizer could be used [11]. Superfine meltblown nonwoven has lots of possibilities in the research areas and some other applications including filtration membranes, medical and health supplies such as bandage mask, oil absorption materials, etc [6] [11] [12].

On the other hand, Thermoplastic Polyurethane (TPU) is a polymer that has been widely used to increase the flexibility and strength of plastics like PLA, polypropylene and some other brittle polymers [13]. The TPU polymer comes 
with several attractive properties such as high strength and toughness, biocompatibility, flexibility, durability, and bio-stability. On the other hand, TPU is a superior candidate for thermoplastic block linear copolymer with tensile strength up to $50 \mathrm{MPa}$ and elongation at break of above 500\% [6] [14]. Excessive addition of TPU in the PLA could decrease the tensile strength of the composite nonwoven [5] [11]. A lot of studies have been effectively employed to improve the toughness properties of PLA and among them, polymer blending is an economical and practical approach for toughening the PLA; moreover, this process is also easy to be industrialized [15]. In order to get rid of these drawbacks, researchers used some compatibilizers [11] such as free radical initiator [16], 1,4Phenylene Diisocyanate (PDI) [17] and maleic anhydride grafted PLA (PLA-gMA) during the meltblown process to improve the PLA/TPU composite nonwoven's interfacial property [18].

For ensuring the security and safety issues, Kanebo Co., Ltd. used TPU meltblown nonwovens for producing work gloves with smooth surface, high heat resistance and dirt resistance [6]. The Donghua University of China blended PLA and Thermoplastic Silicone Polyurethane Elastomer pellets together for producing high mechanical properties of PLA/TPSiU blends the impact strength reached $19.3 \mathrm{~kJ} / \mathrm{m}^{2}$ [19].

Joncryl ADR 4400 used in this study is a compatibilizer. Joncryl ADR4400 is a polymeric chain extender with a medium epoxy equivalent weight (medium number of epoxy groups per chain) that reacts with the chain ends of polycondensates and effectively increases their melt viscosity. It can also be used for the modification of other thermoplastics such as PC, PC/ABS, PBT, TPU, etc. It is a patented, multi-functional reactive polymer with improved thermal stability versus earlier chain extenders, making it a better additive for specific food contact applications based on polycondensation polymers such as PLA, PET, etc [20]. Current study results for improving the miscibility and tensile strength are same as the research done by M.S. Mahmuda for toughening the PLA with TPU by using EMA-GMA compatibilizer [21].

The manuscript proposed a novel model to predict tensile strength and the overall stress-strain dependence of PLA/TPU meltblown fiber mats. Almost no studies focused on PLA/TPU meltblown nanofiber. The corresponding PLA/TPU MB nonwovens were successfully manufactured and the surface morphology, mechanical properties and rheological properties were investigated. The environmentfriendly toughened PLA/TPU meltblown nonwoven was considered to be well applied in the area of disposal filtration such as mask, adsorbing, medical and health care applications.

\section{Experiments}

\subsection{Material}

PLA (grade 6252D especially for MB processing) was supplied by NatureWorks LLC (USA) \& the flexible Thermoplastic Polyurethane (grade was purchased from 
BASF. Joncryl ADR 4400 used in this experiment were also purchased from BASF. All materials were used as received to produce biodegradable melt blown nonwoven without any further purification. The materials used in the experiment all were AR grade and used as received.

\subsection{Material Processing (Melt Blending)}

Melt compounding was carried out via a twin screw extruder (TSE-30A; Nanjing Ruiya Extrusion System Limited, China; length/diameter $[\mathrm{L} / \mathrm{D}]=40$ ) at with a screw diameter of $18 \mathrm{~mm}$ And an L/D ratio of 40 . Prior to melt compounding, the PLA $\&$ TPU were dried in a vacuum oven at $80^{\circ} \mathrm{C}$ for 16 hours non-disturbed to eliminate the moisture. Figure 1 shows that, the PLA/TPU blend in a certain weight ratio of $95 / 5,90 / 10,85 / 15,82 / 20$ was melt mixed with Joncryl ADR 4400 (1\% wt. \% of the composites) in a mixer for 5 minutes. The melt blended extruder 7 chambers temperatures were set at $170^{\circ} \mathrm{C}, 190^{\circ} \mathrm{C}, 200^{\circ} \mathrm{C}, 190^{\circ} \mathrm{C}, 180^{\circ} \mathrm{C}, 180^{\circ} \mathrm{C}$ and $170^{\circ} \mathrm{C}$ respectively.

The extruded blended samples was cooled in a clean normal water bath and then pelletized. The produced pellets were dried in a vacuum oven at $80^{\circ} \mathrm{C}$ for 12 hours undisturbed and afterward stored in a vacuum at room temperature for further processes.

\subsection{Method of Meltblown Preparation}

PLA/TPU meltblown nonwovens were prepared by using an experimental meltblown machine (Jiaxing Longman Measurement and Control Technology Co., Ltd., China). Before doing the meltblown process the blend PLA/TPU was kept in vacuum oven at $80^{\circ} \mathrm{C}$ degree Celsius for 24 hours to remove the moisture. A typical meltblown spinning process and the fixed meltblown parameters in this study are shown in Figure 2. PLA/TPU MB nonwoven were manufactured by a laboratory scale $\mathrm{MB}$ nonwoven machine with a single orifice die. The diameter of the orifice was $0.3 \mathrm{~mm}$; the L/D was 40 . PLA and PLA/TPU
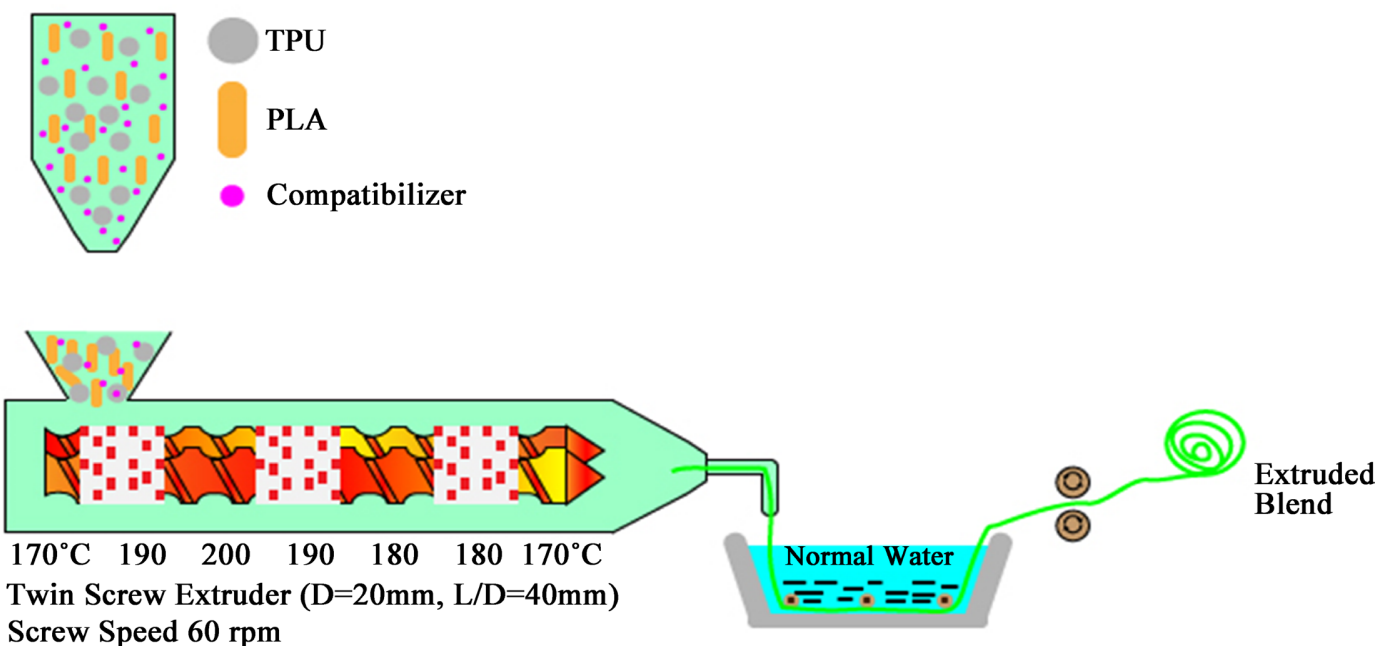

Figure 1. Melt blending of PLA-TPU \& compatibilizer. 


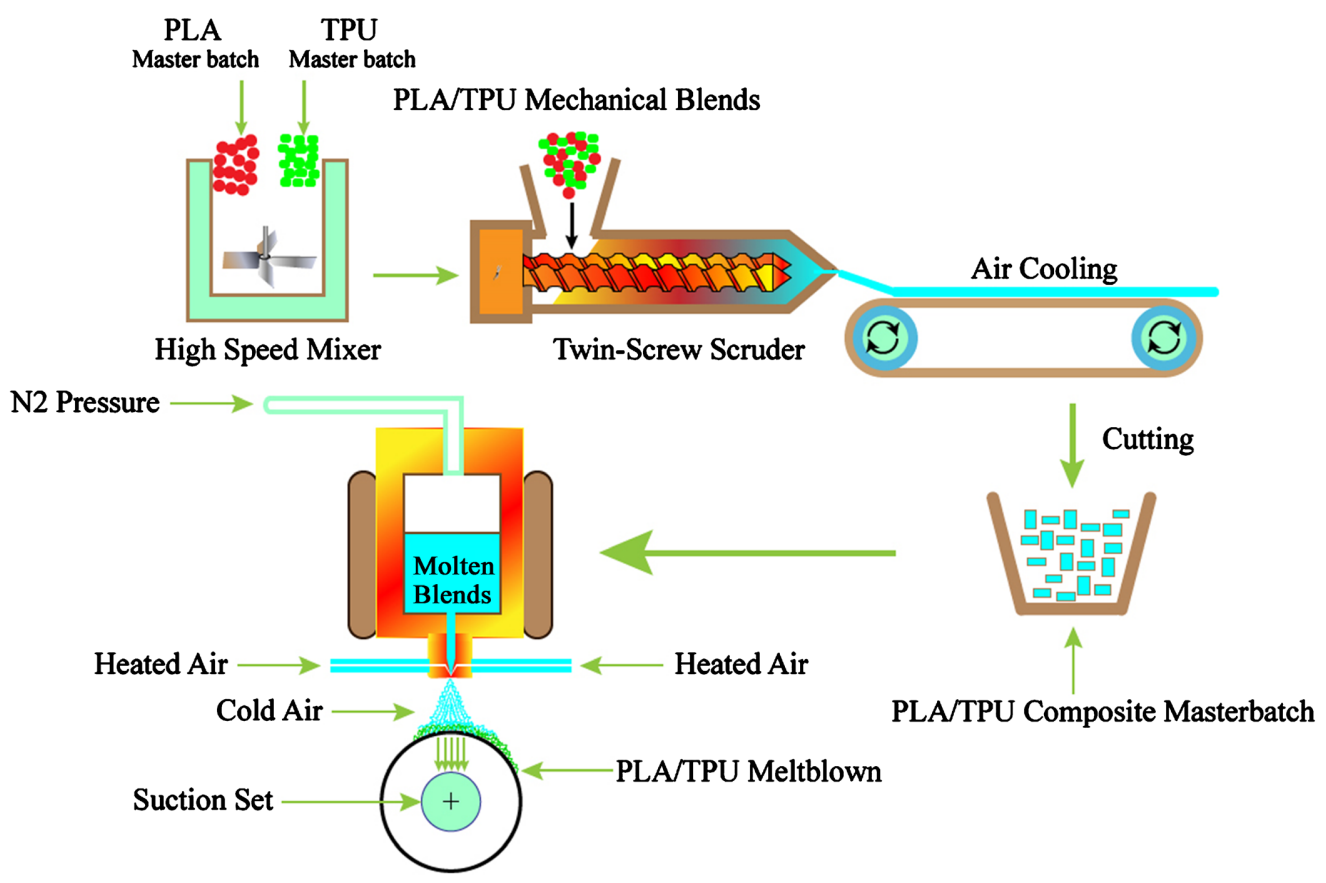

Processing flow of illustrated PLA/TPU Meltblown Composite

Figure 2. Processing flow of PLA/TPU meltblown.

blends were extruded at $240^{\circ} \mathrm{C}-250^{\circ} \mathrm{C}$. Nitrogen gas was injected at a fixed pressure of 0.2 MPa to get a standard and uniform melt flow from the die orifice. The drawing hot air $(0.3 \mathrm{MPa})$ was also kept at $270^{\circ} \mathrm{C}-280^{\circ} \mathrm{C}$. The spun PLA/TPU meltblown fiber were cooled at room temperature and collected on a stainless steel roller (collector) with a suction set; the collector speed and the Die to Collector Distance (DCD) were optimized according to the practical adjustment to get superior quality of meltblown nonwovens fabric.

\subsection{Characterization}

\subsubsection{Differential Scanning Calorimetry (DSC)}

DSC tests were carried out using a Perkin-Elmer DSC 8000 (USA) under N2 atmosphere $(20 \mathrm{~mL} / \mathrm{min})$. Samples of $5 \pm 0.5 \mathrm{mg}$ were weighted and sealed in aluminum crucibles. Samples were heated from $25^{\circ} \mathrm{C}$ to $230^{\circ} \mathrm{C}$ at $10^{\circ} \mathrm{C} / \mathrm{min}$ (first heating), equilibrated at $230^{\circ} \mathrm{C}$ for $3 \mathrm{~min}$ to eliminate the thermal history, cooled to $25^{\circ} \mathrm{C}$ at $10^{\circ} \mathrm{C} / \mathrm{min}$, and then heated again from $25^{\circ} \mathrm{C}$ to $230^{\circ} \mathrm{C}$ at $10^{\circ} \mathrm{C} / \mathrm{min}$ (second heating). The crystal weight fraction $(X c)$ of PLA could be calculated by using Equations from Zhu et al. [22].

$$
X_{c}(P L A)=\frac{\Delta H_{m}(P L A)-\Delta H_{c c}(P L A)}{\Delta H_{0}(P L A)} \times \frac{100}{W_{t}}
$$

Here, $\Delta H_{m}$ is the measured melting enthalpy, $\Delta H_{c c}$ is the measured cold crystallization enthalpy, $\Delta H_{0}$ is the melting enthalpy for $100 \%$ crystalline PLA (93.1 $\mathrm{J} / \mathrm{g})$. 


\subsubsection{Scanning Electron Microscopy of PLA-TPU Blends (SEM)}

The surface morphology and structure of PLA-TPU melt blended polymer with and without Joncryl ADR 4400 observed by using field emission scanning electron microscopy (FE-SEM) (Ultra 55, Carl Zeiss, AG, Germany) at an accelerating voltage of $2-3 \mathrm{kV}$. The specimens were firstly dispersed in liquid nitrogen about 3 - 5 minutes and were cut the sample with two stainless steel handle into small part to attach into the gold plate for the test. A clean and smooth surface plays crucial part to get the good quality images.

\subsubsection{Thermogravimetric Analysis (TGA)}

TGA test was performed using a Mettler Toledo TGA/DSC1/1600 (Switzerland) under the $\mathrm{N} 2$ atmosphere $(20 \mathrm{~mL} / \mathrm{min})$. Samples of $5 \pm 0.5 \mathrm{mg}$ were weighted and put into a ceramic crucible; samples were heated from room temperature to $550^{\circ} \mathrm{C}$ at $20^{\circ} \mathrm{C} / \mathrm{min}$.

\subsubsection{Crystal Forms of Melt Blended PLA/TPU (XRD)}

A Wide-Angle X-Ray Diffractometer (WAXD) was obtained by using a Bruker AXS D8 Discovery (Germany) at room temperature. The PLA, TPU, and MBs samples of $10 \mathrm{~mm} \times 10 \mathrm{~mm}$ were tested. The CuKa radiation source was operated at 40 $\mathrm{kV}$ power and $40 \mathrm{~mA}$ current. The scanning angle ranged from $10^{\circ}$ to $40^{\circ}$ with increments of $0.02^{\circ}$.

\subsubsection{Mechanical Properties of PLA/TPU Blends}

Mechanical behaviors of PLA and PLA/TPU MB nonwovens were investigated using a universal tensile machine (Instron-3369, USA) according to the method of ISO 9073-3-1989. All samples were conditioned under standard laboratory conditions $\left(25^{\circ} \mathrm{C} \pm 2{ }^{\circ} \mathrm{C}\right.$ and $\left.65 \% \mathrm{RH}\right)$ for $24 \mathrm{~h}$. Samples with the size of $50 \mathrm{~mm}$ $20 \mathrm{~mm}$ were selected in rolling direction, and the tensile speed was $50 \mathrm{~mm} / \mathrm{min}$. All the results were calculated as the average of five specimens.

\subsubsection{Rheology of PLA/TPU Blends}

Rheological behaviors were measured using a twin column capillary rheometer (Rosand RH7, UK) with a length-to diameter ratio (L/D) of 16 and the diameter of $1 \mathrm{~mm}$. Test began with two times of pre-compression (pressure $0.3 \mathrm{MPa}$ ) and preheat (a total of $3 \mathrm{~min}$ ) to ensure that the samples were melt and compacted, and the temperatures were set at $210^{\circ} \mathrm{C}$. Shear stress and shear rate were corrected using a zero-length die.

\section{Result \& Discussion}

\subsection{Melting \& Crystallization: DSC}

DSC second heating and cooling curves of PLA/TPU blends are shown in Figure 3, and the corresponding thermal parameters are listed in Table 1. In the trace of PLA heating curve, the glass transition temperature $(\mathrm{Tg})$ was $57.71^{\circ} \mathrm{C}$ and the cold crystallization temperature (Tcc) at $111.89^{\circ} \mathrm{C}$ was observed due to 


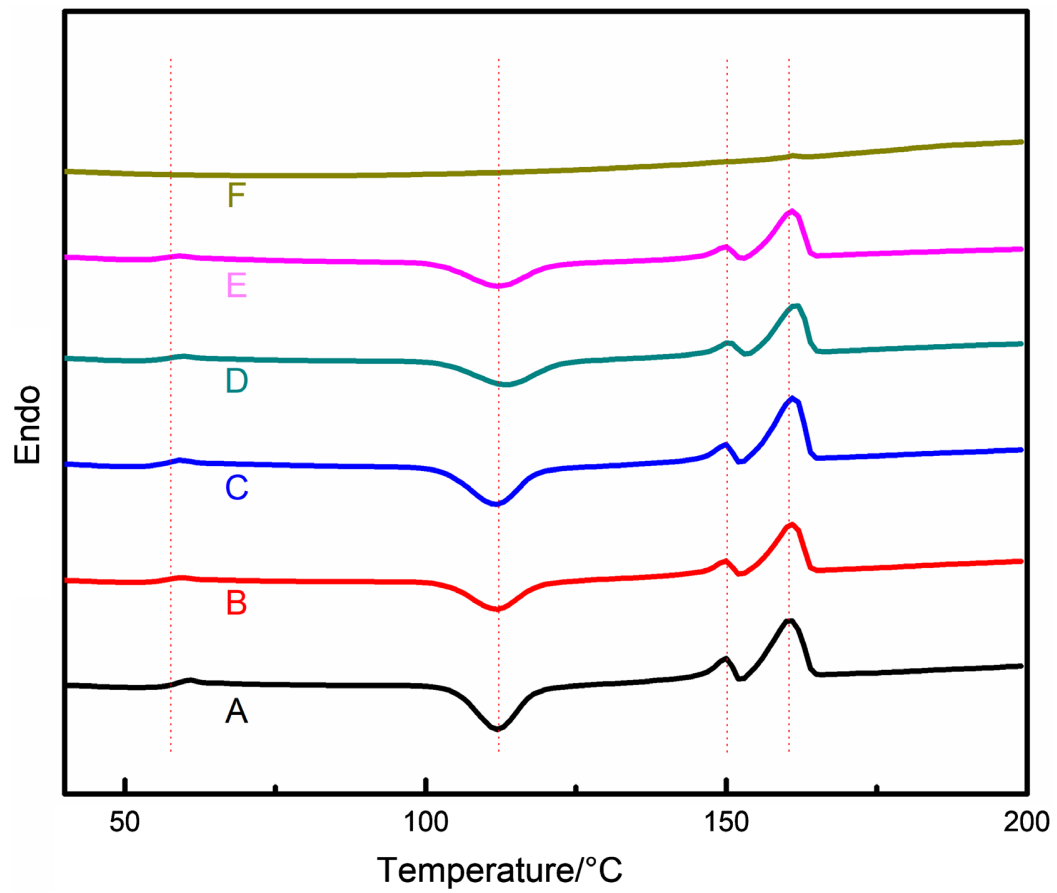

Figure 3. DSC heating curves of PLA/TPU blends: (A) 100/0, (B) 95/5, (C) 90/10, (D) 85/15, (E) 80/20, (F) TPU.

Table 1. PLA TPU DSC heating rate.

\begin{tabular}{cccc}
\hline Pla/Tpu (wt\%) & $T_{g}$ (PLA) & $T_{c c}$ (PLA) & $T_{m 1}$ (PLA) \\
\hline $100 / 0$ & 57.71 & 111.89 & 150.11 \\
$95 / 5$ & 57.51 & 112.23 & 150.39 \\
$90 / 10$ & 57.52 & 111.77 & 149.77 \\
$85 / 15$ & 57.85 & 112.84 & 150.89 \\
$80 / 20$ & 57.24 & 112.13 & 150.39 \\
TPU & - & - & -
\end{tabular}

the mobility and rearrangement of PLA macromolecules.

In Table 1, PLA displayed two obvious separated melting peaks (Tm) which could be explained by the mechanisms of melt recrystallization, multiple lamellae, or crystal structures. In the cooling process of PLA, no obvious crystallization peak $(\mathrm{Tc})$ was found because of its slow crystallization rate.

\subsection{Scanning Electron Microscopy (SEM) of Poly (Lactic Acid) \& Thermoplastic Poly Urethane}

The morphology of composites was observed by scanning electron microscopy (SEM). Figure 4 shows the SEM images of the cryofractured surface of neat polymers, non-compatibilized and compatibilized PLA/TPU blends with various weight compositions. Two important surface morphologies can be expected such as sea-island structure or matrix-droplet morphology and a co-continuous 
morphology when two or more immiscible polymers were melt blended together [21] [23]. SEM images (A-B-C-D-E) shows the morphological structure of the PLA/TPU 100/0, 95/5, 90/10, 85/15 and 80/20 respectively. The development of phase morphology significantly affects the properties of polymer blends and this morphological development was influenced by the different blend ratios and compositions. Adding an additive or compatibilizer can plays a vital role to make this change. All of the blends show a clear, phase-separated morphology with TPU dispersed in the PLA matrix. It can be seen in Figure 4 (image A) that $100 \%$ melt blended PLA has a smooth fractured and homogeneous surface comparatively to other blended polymer surfaces even after adding the compatibilizer. The phase morphology in binary PLA/TPU polymer blends was generally affected by the weight and viscosity ratios of blending components. It was obvious to find that PLA/TPU blends still presented a "sea-islands" structure as the blends images showed. TPU phases were uniformly dispersed in PLA (Poly (Lactic Acid)) matrix as globules shape roughly $8-9 \mathrm{~mm}$ in diameters, and some dispersed phases began to contact and even compress with each other when increasing the blending weight ratio of TPU. However, the interface between PLA and TPU phases in PLA/TPU/joncryl adr 4400 blends was effectively intensified in comparison to the PLA/TPU blends reported in previous studies [24]. It should be noted that some voids and de-bonding still existed, and the interface between PLA and TPU was not completely "wetted" by the compatibilizer, especially when the blending proportion of joncryl adr 4400 was low, i.e., PLA/TPU/joncryl adr 4400 (95/5/1). It was observed that, as the amount of TPU was increased in the composites, the
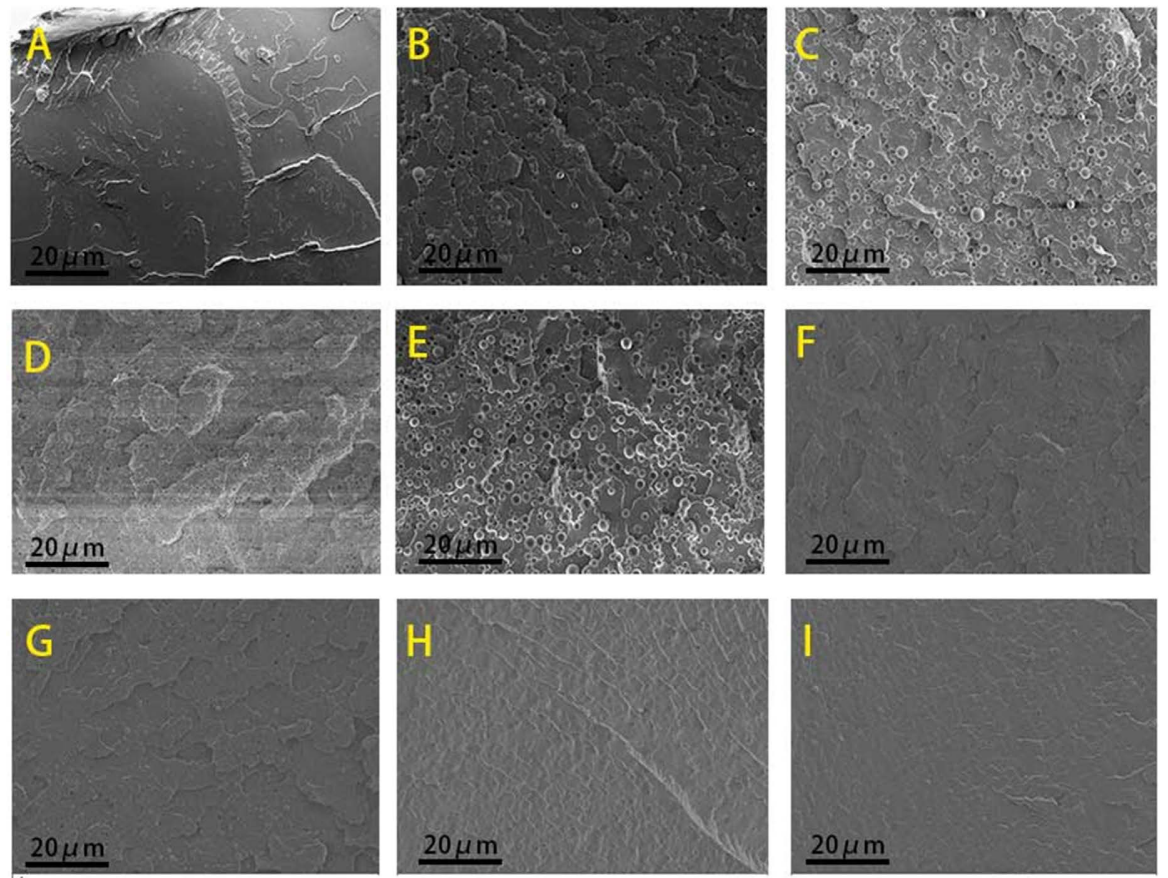

Figure 4. SEM images of PLA/TPU melt blending with and without compatibilizer: (A) 100/0 (B) 95/5, (C) 90/10, (D) 85/15, (E) 80/20 (F) 95/5/1 (G) 90/10/1 (H) 85/15/1 (I) $80 / 20 / 1$. 
thermal stability and flexibility increased; on the other hand the brittleness of PLA reduced. The glass transition temperatures of the composites were increased with the addition of thermoplastic polyurethane and it could be attributed due to the decrease in the segmental motion of the polymer chains.

\subsection{Thermal Properties (Thermo Gravimetric Analysis)}

As PLA is a semicrystalline polymer, and its mechanical properties strongly depend on its crystallization behavior. Biodegradable polymers such as PLA, polycaprolactone, polybutylene succinate, polybutylene succinate adipate, etc. are generally displayed poor thermal stability, which affected the processing and properties of end product [11]. Polymeric and composite materials properties such as thermal stability and glass transition temperature $(\mathrm{Tg})$ are very important parameters for the composite materials applications. Figure 5 shows the weight as a function of temperature for PLA/TPU blends with and without compatibilizer. A single thermal degradation stage was observed in both PLA and TPU.

Figure 5 showed that, the initial thermal decomposition temperature $\left(\mathrm{T}_{\text {initial }}\right)$ of PLA was about $280.31^{\circ} \mathrm{C}$, and the practical MB processing temperature was generally $<250^{\circ} \mathrm{C}$. In comparison with PLA, TPU displayed higher thermal stability with the ( $\left.\mathrm{T}_{\text {initial }}\right)$ of $333.6^{\circ} \mathrm{C}$. The thermal degradation of PLA and TPU took place via different mechanisms, since two obvious decomposition stages could be observed in PLA/TPU blends. Both non-free and free radical theories were adopted to explain the thermal degradation of PLA [11] [22]. The glass transition of Poly (Lactic Acid) (PLA) is considerably affected by the addition of TPU and on the other hand glass transition of TPU phase changes very little with the addition of PLA.

\subsection{X-Ray Diffraction Analysis}

\section{Crystal Forms of Melt Blended PLA/TPU}

To investigate the effect of TPU on the crystalline structure of PLA, the WAXD patterns of TPU with and without modification and PLA/TPU MBs were determined, as shown in Figure 6. The XRD graph shows that all the base lines of the melt blended pure PLA and PLA/TPU were nearly the same. Only two major pea $\mathrm{k} \mathrm{s}$ found in the XRD graph. The main two peaks of PLA blend were found at $2 \theta$ (theta) angle of $16.64^{\circ}$ and $18.94^{\circ}$ which corresponds to $110 \& 203$ lattice plane respectively and another two peak s found at $14.75^{\circ} \& 22.31^{\circ}$ which corresponds to (010) \& (15) lattice plane respectively [25]. Adding TPU in the PLA there were no significant differences in the peak values, suggesting that TPU did not affect the basic structure of PLA [26].

The low crystallinity degree of Poly (Lactic Acid) (PLA) MBs could be attributed to two main reasons such as: 1) the crystallization rate of PLA was inherently low at room temperature; and the 2) PLA meltblown fibers did not have enough time to be crystallized during the rapid production process of melt blowing because it requires lower temperature to be melted [27]. 


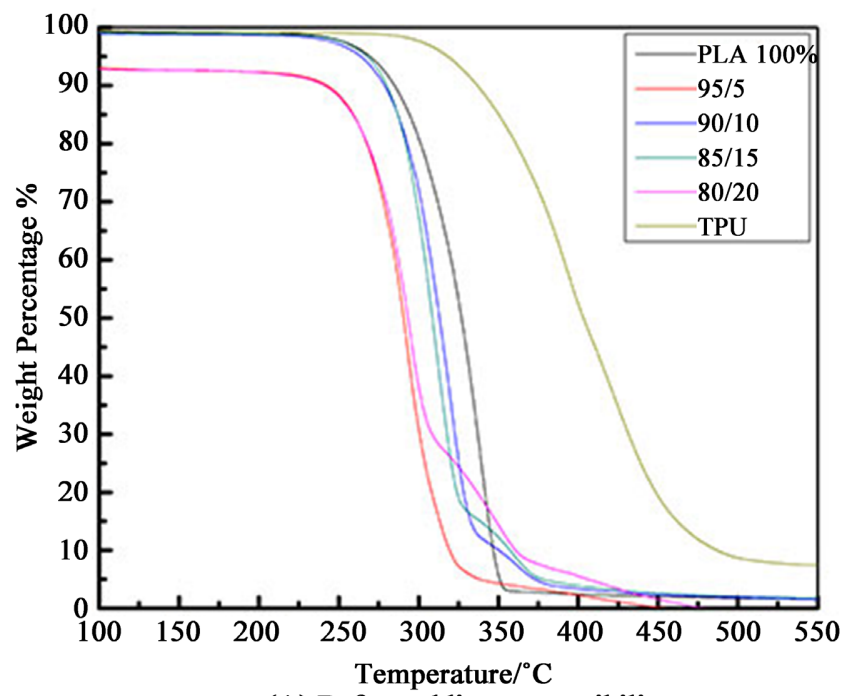

(A) Before adding compatibilizer

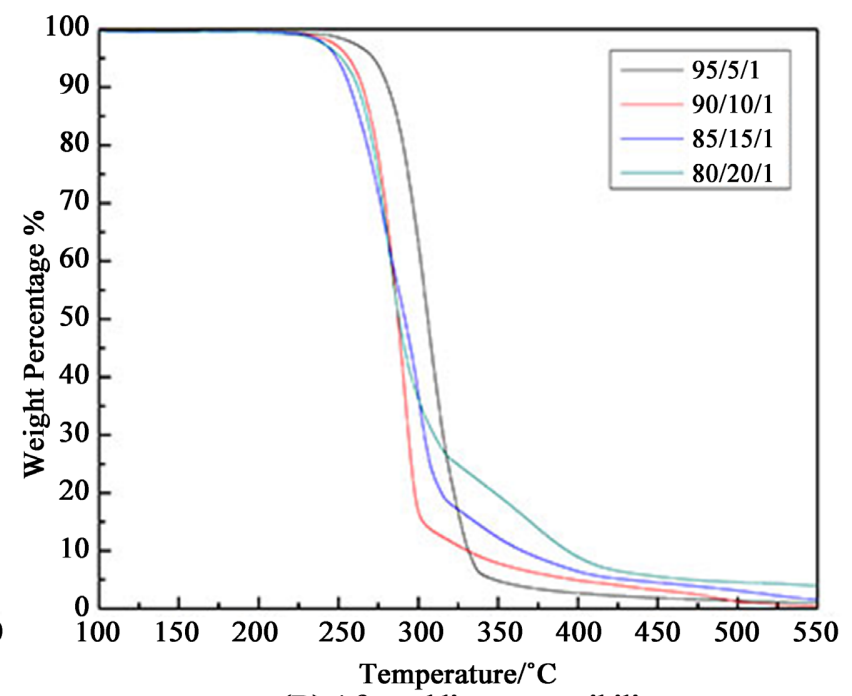

(B) After adding compatibilizer

Figure 5. TG curves of PLA/TPU blends before and after adding the compatibilizer: In picture (A): (a) 100\% PLA; (b) 95/5; (c) 90/10; (d) 85/15; (e) 80/20; (f) TPU. In picture (B): (a) 95/5/1; (b) 90/10/1; (c) 85/15/1; (d) $80 / 20 / 1$.

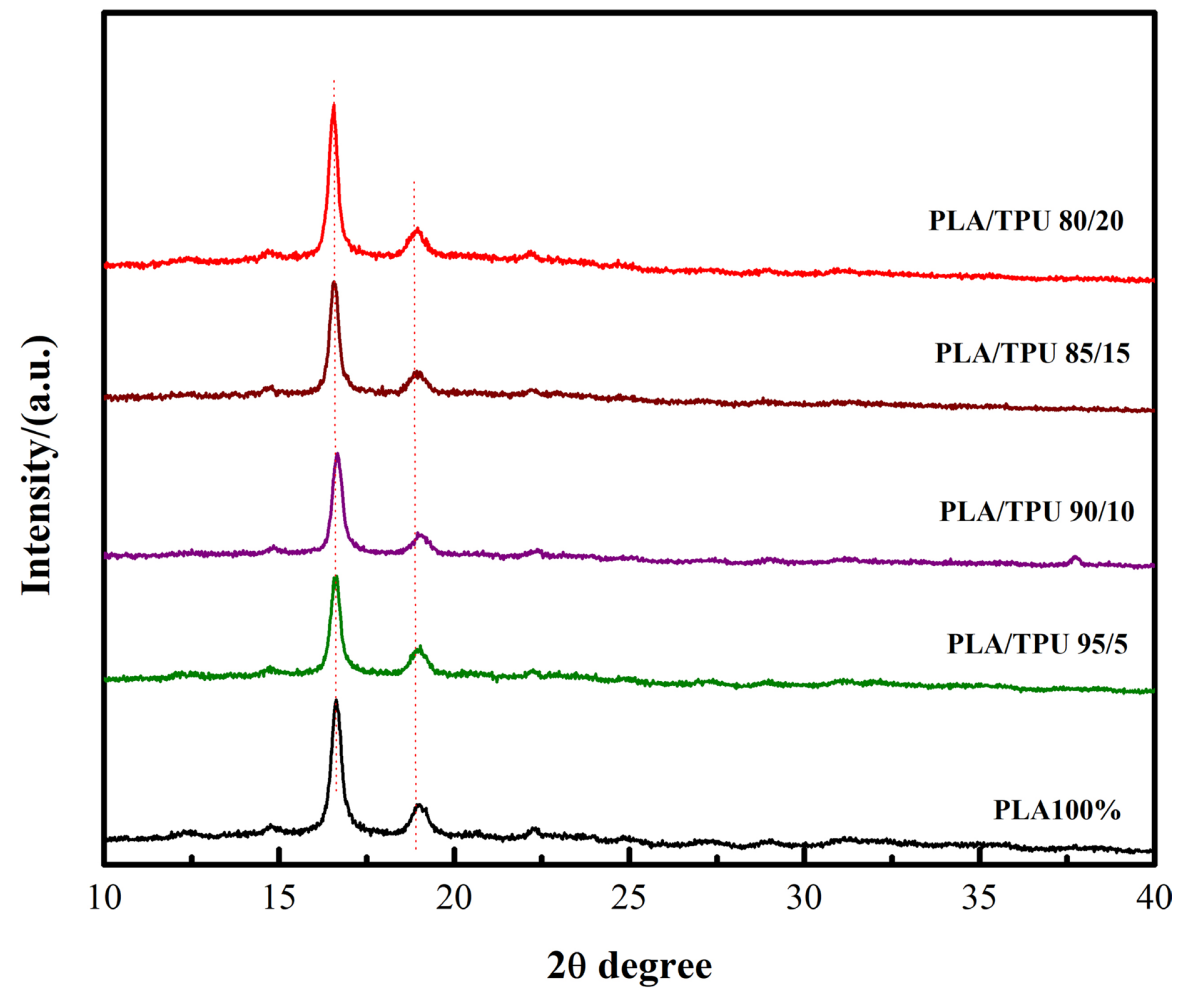

Figure 6. Wide-angle X-ray diffractometer patterns melt blended PLA/TPU: (a) 100/0; (b) $95 / 5$; (c) $90 / 10$; (d) $85 / 15$; (e) $80 / 20$.

\subsection{Mechanical Properties of PLA/TPU Composite Nonwoven (Ten- sile)}

The tensile mechanical properties of PLA/TPU meltblown nonwovens are shown in Figure 7. Basically the meltblown is an advanced process that can produce high quality nonwovens fabric with porous and fluffy structures (nanofiber) 

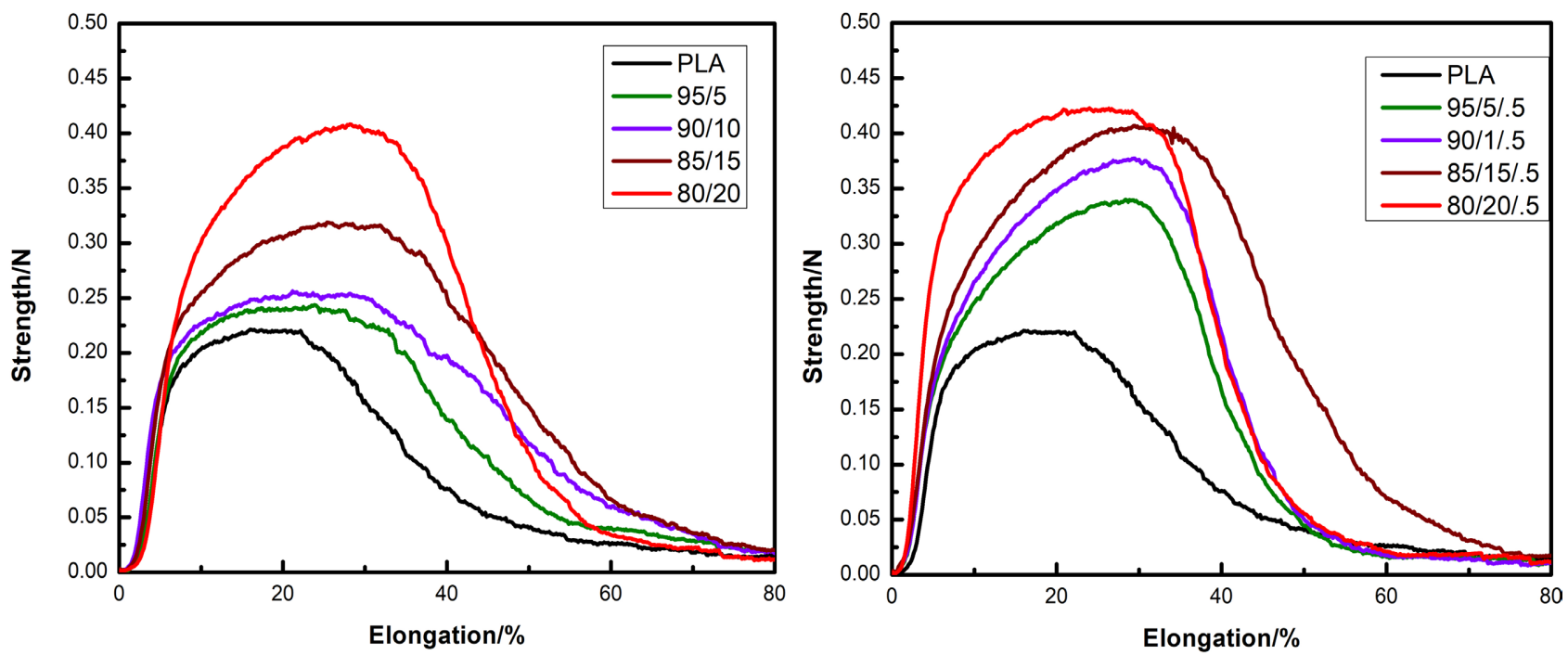

Figure 7. Tensile strength elongation curves of PLA/TPU MB nonwovens after adding compatibilizer.

fiber), consist of many superfine fibers and cohesive points which provide the mechanical strength in the fabric. When the meltblown nonwovens started stretching, generally the tensile force first makes the nonwoven fabric get narrower and gradually the fiber's strength falls down at a $\mathrm{n}$ equilibrium stage. The addition of TPU at $15 \%$ and $20 \%$ could generate a noticeable improvement in the toughness of the poly (lactic acid), as figured out in the figure above in accordance with enhanced elongation at break especially, at $15 \mathrm{wt} \%$ content. In the above figure, it showed that PLA/TPU 85/15 linear curve 0.40 Mpa exhibits higher tensile strength compared to pure PLA or others blend. This means that with the addition of TPU, even though only $5 \%$, the spinnability and tensile properties of the material is obviously decreased. Beside the last composite 80/20 although strength is higher but the durability and elongation at break point very closer to low amount of TPU added percentages. In this research it found that inappropriate amount of adding TPU or an additive resulting in poor or irregular tensile strength.

\subsection{Rheological Behaviors}

Based on the two curves above it has been seen that the viscosity is increasing gradually as the amount of TPU is increasing in the PLA blend. The rheological properties of PLA/TPU blends are investigated at $200^{\circ} \mathrm{C}$ and $210^{\circ} \mathrm{C}$ temperatures. Capillary Rheological Characterization melt blended at a certain shear rate dynamic apparent viscosity size. In this study, small amplitude oscillatory shear rheological experiments were conducted to compare the melt viscoelastic behaviors of the neat PLAs and the blends and to assess the compatibility between the PLA and TPU phases.

Figure 8(A) and Figure 8(B) show PLA/TPU apparent viscosity of the melt blended material shear rate increases and decreases. When the shear rate is near the $0 \mathrm{rad} / \mathrm{s}$, at the beginning the apparent viscosity of the melt with the rise in 

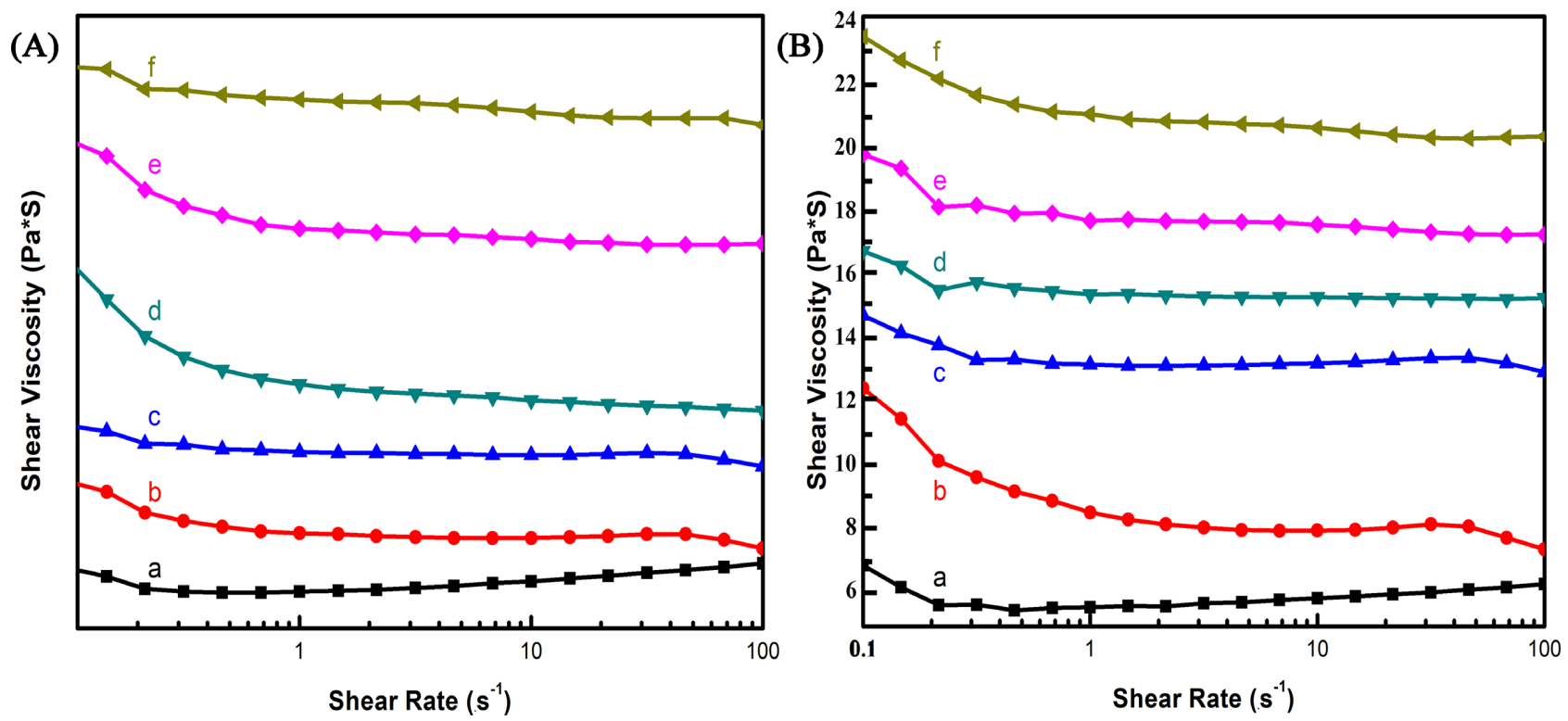

Figure 8. Apparent viscosity $\eta$ a versus shear rate $\gamma$ at $200^{\circ} \mathrm{C} \& 210^{\circ} \mathrm{C}$ of PLA/TPU blends: (a) 100/0; (b) $95 / 5$; (c) $90 / 10$; (d) $85 / 15$; (e) $80 / 20$; (f) $0 / 100$.

the mass ratio of TPU is slightly increased. Due to the TPU the viscosity of the blends increases which may be ascribed to the higher viscosity of TPU than that of PLA under the processing temperature. However, the range of blend viscosity increase is still limited. At the same time, the addition of catalyst has no obvious influence on the apparent viscosity of blends. Overall at two different temperatures are used to understand the PLA/TPU blends rheology properties. As the temperature increases the apparent viscosity of the sample has decreased. With the change in the shear rate the apparent viscosity curve substantially similar for all samples. The increasing of temperatures the shear viscosity decreased because of macromolecules chain movement enhanced so that apparent viscosity is also decreased. Increasing the rate of apparent viscosity is because of the entanglement PLA and TPU chains molecules. The entanglement between molecular chains of PLA and TPU is reduced which cause the reduction of the apparent viscosity.

\section{Conclusion}

The applications of meltblown nonwovens fabrics have continually been growing. In the future, meltblown products will continue to increase rapidly in market share and can easily penetrate new markets including some portions of the apparel market. Poly (Lactic Acid) and Thermoplastic Polyurethane blends were fabricated by melt blowing process to prepare a biodegradable composite material with improved toughed quality. A novel model was developed to predict tensile strength and the overall stress-strain dependence of meltblown fiber mats. The corresponding PLA/TPU MB nonwovens were successfully manufactured and the surface morphology and mechanical properties were investigated. DSC results revealed that the addition of TPU elastomer not only accelerates the crystal- 
lization rate of PLA, but also decreases the PLA crystallinity. The tensile strength and elongation of PLA/TPU MB nonwovens were improved, among which the strength and the elongation at the maximum strength of PLA/TPU (85/15) MB nonwovens were increased in comparison with that of PLA; however, some defects on PLA/TPU MB webs also occurred when the blending proportion of TPU increased to $20 \%$. The environmentally friendly toughened PLA/TPU meltblown nonwoven was considered to be well applied in the area of disposal filtration such as mask, adsorbing, medical and health care applications. However, to improve the quality of MB nonwoven the miscibility between PLA/TPU should be further improved using another additive or applying another method. Further studies should focus on increasing the antibacterial properties by adding some antibacterial agents to get better results.

\section{Conflicts of Interest}

No potential conflicts of interest were reported by the authors.

\section{References}

[1] Nayak, R., Padhye, R., Kyratzis, I.L., Truong, Y.B. and Arnold, L. (2012) Recent Advances in Nanofibre Fabrication Techniques. Textile Research Journal, 82, 129-147. https://doi.org/10.1177/0040517511424524

[2] Tan, D.H., Zhou, C., Ellison, C.J., Kumar, S., Macosko, C.W. and Bates, F.S. (2010) Meltblown Fibers: Influence of Viscosity and Elasticity on Diameter Distribution. Journal of Non-Newtonian Fluid Mechanics, 165, 892-900. https://doi.org/10.1016/j.jnnfm.2010.04.012

[3] Moyo, D., Patanaik, A. and Anandjiwala, R.D. (2012) Process Control in Nonwovens Production. Woodhead Publishing Limited, Sawston. https://doi.org/10.1533/9780857095633.3.279

[4] ASTM International (2004) Standard Test Method for Thickness of Nonwoven Fabrics. ASTM International, West Conshohocken, 1-4.

[5] Ma, Z., Kotaki, M., Yong, T., He, W. and Ramakrishna, S. (2005) Surface Engineering of Electrospun Polyethylene Terephthalate (PET) Nanofibers towards Development of a New Material for Blood Vessel Engineering. Biomaterials, 26, 2527-2536. https://doi.org/10.1016/j.biomaterials.2004.07.026

[6] Peng, M., Jia, H., Jiang, L., Zhou, Y. and Ma, J. (2018) Study on Structure and Property of PP/TPU Melt-Blown Nonwovens. The Journal of the Textile Institute, 110 , 468-475.

[7] Lin, W. and Qu, J.P. (2019) Enhancing Impact Toughness of Renewable Poly(lactic acid)/Thermoplastic Polyurethane Blends via Constructing Cocontinuous-Like Phase Morphology Assisted by Ethylene-Methyl Acrylate-Glycidyl Methacrylate Copolymer. Industrial \& Engineering Chemistry Research, 58, 10894-10907. https://doi.org/10.1021/acs.iecr.9b01644

[8] Castro-Aguirre, E., Iñiguez-Franco, F., Samsudin, H., Fang, X. and Auras, R. (2016) Poly(Lactic Acid)-Mass Production, Processing, Industrial Applications, and End of Life. Advanced Drug Delivery Reviews, 107, 333-366. https://doi.org/10.1016/j.addr.2016.03.010

[9] Santoro, M., Shah, S.R., Walker, J.L. and Mikos, A.G. (2016) Poly(Lactic Acid) Nano- 
fibrous Scaffolds for Tissue Engineering. Advanced Drug Delivery Reviews, 107, 206 212. https://doi.org/10.1016/j.addr.2016.04.019

[10] Dong, Y., Marshall, J., Haroosh, H.J., Moghaddam, S.M., Liu, D., Qi, X. and Lau, K. (2015) Polylactic Acid (PLA)/Halloysite Nanotube (HNT) Composite Mats: Influence of HNT Content and Modification. Composites: Part A, 76, 28-36. https://doi.org/10.1016/j.compositesa.2015.05.011

[11] Md Obaidur Rahman, B. and Zhu, F.C. (2021) Study on Toughened Polylactic Acid and Its Meltblown Nonwovens by Thermoplastic Polyurethane. Silk, 58, 28-35. https://doi.org/10.3969/j.issn.1001-7003.2021.10.006

[12] Sinha-Ray, S., Yarin, A.L. and Pourdeyhimi, B. (2014) Meltblown Fiber Mats and Their Tensile Strength. Polymer (Guildf), 55, 4241-4247.

https://doi.org/10.1016/j.polymer.2014.05.025

[13] Poomalai, P. (2005) Siddaramaiah, Studies on Poly(Methyl Methacrylate) (PMMA) and Thermoplastic Polyurethane (TPU) Blends. Journal of Macromolecular Science Part A Pure and Applied Chemistry, 42, 1399-1407. https://doi.org/10.1080/10601320500205764

[14] Jia, S., Wang, Z., Zhu, Y., Chen, L. and Fu, L. (2015) Composites of Poly(Lactic) Acid/Thermoplastic Polyurethane/Mica with Compatibilizer: Morphology, Miscibility and Interphase. RSC Advances, 5, 98915-98924. https://doi.org/10.1039/C5RA17938F

[15] Sin, L.T. and Tueen, B.S. (2019) Thermal Properties of Poly(Lactic Acid). In: Sin L.T. and Tueen, B.S., Eds., Polylactic Acid: A Practical Guide for the Processing, Manufacturing, and Applications of PLA, Second Edition, Elsevier, Amsterdam, 97-133. https://doi.org/10.1016/B978-0-12-814472-5.00003-0

[16] Zhang, L., Xiong, Z., Shams, S.S., Yu, R., Huang, J., Zhang, R. and Zhu, J. (2015) Free Radical Competitions in Polylactide/Bio-Based Thermoplastic Polyurethane/Free Radical Initiator Ternary Blends and Their Final Properties. Polymer (Guildf), 64, 69-75. https://doi.org/10.1016/j.polymer.2015.03.032

[17] Dogan, S.K., Boyacioglu, S., Kodal, M., Gokce, O. and Ozkoc, G. (2017) Thermally Induced Shape Memory Behavior, Enzymatic Degradation and Biocompatibility of PLA/TPU Blends: "Effects of Compatibilization". Journal of the Mechanical Behavior of Biomedical Materials, 71, 349-361. https://doi.org/10.1016/j.jmbbm.2017.04.001

[18] Kaynak, C. and Meyva, Y. (2014) Use of Maleic Anhydride Compatibilization to Improve Toughness and Other Properties of Polylactide Blended with Thermoplastic Elastomers. Polymers for Advanced Technologies, 25, 1622-1632. https://doi.org/10.1002/pat.3415

[19] Sun, M., Huang, S., Yu, M. and Han, K. (2021) Toughening Modification of Polylactic Acid by Thermoplastic Silicone Polyurethane Elastomer. Polymers (Basel), 13, 1953. https://doi.org/10.3390/polym13121953

[20] Features, K. (2014) Joncryl ${ }^{\oplus}$ Functional Additives Joncryl ${ }^{\circledR}$ ADR 4400 Polymeric Chain Extender for Food Contact Applications.

[21] Mahmuda, M.S., Buysa, Y.F., Anuara, H. and Sopyana, I. (2019) Miscibility, Morphology and Mechanical Properties of Compatibilized Polylactic Acid/Thermoplastic Polyurethane Blends. Materials Today: Proceedings, 17, 778-786. https://doi.org/10.1016/j.matpr.2019.06.362

[22] Zhu, F., Yu, B., Su, J. and Han, J. (2020) Study on PLA/PA11 Bio-Based Toughening Melt-Blown Nonwovens. Autex Research Journal, 20, 24-31.

https://doi.org/10.2478/aut-2019-0002 
[23] Kilic, N.T., Can, B.N., Kodal, M. and Özkoç, G. (2021) Reactive Compatibilization of Biodegradable PLA/TPU Blends via Hybrid Nanoparticle. Progress in Rubber, Plastics and Recycling Technology, 1-26. https://doi.org/10.1177/14777606211019423

[24] Nofar, M. (2020) Effect of TPU Hard Segment Content on the Rheological and Mechanical Properties of PLA/TPU Blends. Journal of Applied Polymer Science, 137, 49387. https://doi.org/10.1002/app.49387

[25] Lai, S.M., Wu, W.L. and Wang, Y.J. (2016) Annealing Effect on the Shape Memory Properties of Polylactic Acid (PLA)/Thermoplastic Polyurethane (TPU) Bio-Based Blends. Journal of Polymer Research, 23, Article No. 99.

https://doi.org/10.1007/s10965-016-0993-6

[26] Ji, X., Gao, F., Geng, Z. and Li, D. (2021) Fabrication of Thermoplastic Polyurethane/Polylactide Shape-Memory Blends with Tunable Optical and Mechanical Properties via a Bilayer Structure Design. Polymer Testing, 97, Article ID: 107135. https://doi.org/10.1016/j.polymertesting.2021.107135

[27] Zhu, F., Su, J., Zhao, Y., Hussain, M., Yasin, S., Yu, B. and Han, J. (2019) Influence of Halloysite Nanotubes on Poly(lactic acid) Melt-Blown Nonwovens Compatibilized by Dual-Monomer Melt-Grafted Poly(lactic acid). Textile Research Journal, 89, 4173-4185. https://doi.org/10.1177/0040517519826926 